\title{
ELECTRONIC EMERGENCY MEDICAL TECHNICIAN REPORTS - TESTING A PERCEPTION OF A PROTOTYPE
}

\author{
Smiljana Cuk, Georgia Southern University, sc07273@georgiasouthern.edu \\ Hayden Wimmer, Georgia Southern University, hwimmer@georgiasouthern.edu \\ Loreen M. Powell, Bloomsburg University, lpowell@bloomu.edu \\ Carl M. Rebman Jr., University of San Diego, carlr@sandiego.edu
}

\begin{abstract}
Emergency Medical Technicians (EMTs) still commonly complete a paper-based report, called a quick reference sheet (QRS) or patient care report (PCR) when they are providing services to patients in route to a medical facility. The paper-based report suffers from many challenges such as being lost during patient hand-off, difficulty writing in a moving emergency vehicle, and duplication of information entry. In order to address the aforementioned challenges, we take a first step toward developing a prototype electronic quick reference sheet. Our prototype was built using the Universal Windows Platform to ensure cross-device compatibility. Nineteen emergency medical technicians (EMT) participated in a test of the prototype. The EMT participants were asked to provide feedback on the use of paperbased versus electronic quick reference sheets. The results indicate that EMT's prefer the electronic report. Results of this study found that EMTs perceived electronic Quick Reference Sheets as a better way of collecting the information, easier to complete, and a more efficient way of delivering the information to the hospital.
\end{abstract}

Keywords: Healthcare Informatics, Electronic Medical Records, Medical Information Systems, Quick Reference Sheet, Patient Care Report, Data Transfer, Patient Handover, Emergency Medical Technicians

\section{INTRODUCTION}

According to the Census Bureau (CB17-100), the US nation's population has a distinctly older age profile that it did 16 years ago and the median age is increasing in most areas of the country. The medical care of this aging population is one of today's greatest concerns facing the healthcare sector (NSH Confederation, 2008; Poulymenopoulou, Malamateniou, \& Vassilacopoulos, 2014). As a result, there has been a substantial growth in requests for emergency pre-hospital and in-hospital services, which can benefit from the support from the information exchange of data within an electronic form (Poulymenopoulou et al., 2014).

Moreover, the rapid advancement of electronic medical data to be used for documentation of patient conditions and treatments has also dramatically increased over the last decade from the Affordable Care Act (2008). The Affordable Care Act (2008) authorized electronic medical records (EMS) throughout the United States (US). While the landscape of in-hospital (secondary and tertiary care) as well as primary care has adopted electronic accounts, the pre-hospital sector is slow to implement or integrate consistent electronic records (Andreasson \& Winge, 2010; Cuk, Wimmer, \& Powell, 2017; Finnell \& Overhage, 2010; Frellick \& Vega, 2016; Poulymenopoulou et al., 2014; Rigby, Hill, Koch, \& Keeling, 2011; Rizk, 2010). Currently, a majority of emergency medical technicians (EMT's) throughout the United States use paper Quick Reference Sheets with documenting and handing off the patient to in-hospital, emergency personnel(Cuk et al., 2017; Starmer et al., 2014). Despite the limited number of EMTs and ambulance crews using EMS, the results are positive (Baird \& Boak, 2016; Bledsoe, Wasden, \& Johnson, 2013; Newgard, Zive, Jui, Weathers, \& Daya, 2012). Internationally, many EMTs are also finding positive outcomes from integrating EMS (Johnson, Fraser, Wyatt, \& Walley, 2014; Sheikh, Jha, Cresswell, Greaves, \& Bates, 2014; Zorab, Robinson, \& Endacott, 2015). However, a majority of EMTs still use paper Quick Reference sheets when assessing the patient's needs and condition for a proper hand-off to emergency room personnel (Cuk et al., 2017; Poulymenopoulou et al., 2014).

There is little research into the processes of EMT adoption of EMS. The purpose of this study is to design a prototype of electronic Quick Reference Sheet for EMTs and compare their perceptions regarding usage, completion, collection, and efficiency of data between the paper and electronic Quick Reference Sheets. This work has practical implications for the health informatics field, EMTs and patients. The remaining structure of this paper is as follows. First is a review 
Issues in Information Systems

Volume 19, Issue 3, pp. 81-91, 2018

of the relevant literature followed by stating our hypotheses and methodology. This discussion is followed with the results of our survey. The last section discusses limitations and future directions.

\section{LITERATURE REVIEW}

Healthcare within the US has changed due to rising costs, EMS, and the large aging population (Poulymenopoulou et al., 2014). Currently, many in-hospital (secondary and tertiary care) as well as primary care providers have adopted EMS and electronic medical records (EMR) to aid the healthcare of patients as they moved from one doctor to a specialist and vice-versa. Additionally, EMS and EMR have helped with the research examination of data (data science) for finding patterns, associations, and cures. However, the usage of electronic data or forms has not seen such growth in the pre-hospital level.

Currently, less than a half of EMTs do not collect or use electronic sheets, reports or data (Landman et al 2012)., Cuk et al. (2017), argued that there is needed improvement in the electronic integration of data and forms for EMTs to use during the pre-hospital phase. They believe that integrating EMS with ambulances should be high importance as EMS may improve patients' outcomes. Additionally, Baird and Boak (2016) have found that pre-hospital assessment and documentation impact the success of a patients care within the in hospital transfer.

There are several factors driving adoption of EMS throughout the healthcare systems. Some of the factors are the cost reduction, improved quality of care through availability of patient information(Kilmon, Fagan, Pandey, \& Belt, 2008), performing quality assurance reports easier, faster data transfer, and improved patients' outcomes (Car, 2008; Kilmon et al., 2008). Cuk et al. (2017) examined EMTs' perceptions regarding the EMS, forms and sheets. Their study revealed that EMTs are favorable EMS, sheets or forms. EMTs liked to see a change from paper to electronic transfer of information. Still, very slow change is occurring regarding the usage electronic data or forms in the pre-hospital sector.

There are factors that may explain the slow growth in EMS within the pre-hospital or EMTs sector. Landman, Lee, Sasson, Van Gelder, and Curry (2012) performed a qualitative research study regarding EMT's perceptions regarding the adoption of EMS or electronic sheets/forms. Their study listed the following reasons as challenges to EMS adoption as quality assurance, increased run times due to transitioning to a new system, lack of integration of e-PCR systems with existing hospital information systems, and deficiency of funding.

Another factor contributing to the slow growth in electronic data and forms within the pre-hospital sector is healthcare professionals' perceived ease of use of information technology (Maillet, Mathieu, \& Sicotte, 2015; Money et al., 2015). While the ease of use or adoption of EMS within the physician sector of the healthcare system has been widely studied (Audet, Squires, \& Doty, 2014; Hamid \& Cline, 2013; Jensen \& Aanestad, 2006; Rivard \& Lapointe, 2012), it has not been widely studied from an EMT perspective. Consequently, there is limited literature on the EMTs and other emergency personnel's adoption of EMS within the US.

\section{RESEARCH HYPOTHESES}

In an effort to contribute to the limited EMT research regarding EMS, this research study seeks to design and develop an electronic Quick Reference Sheet and compare it to the current paper version. The goal of this research study is to compare EMT's and emergency personnel's perceptions regarding usage, completion, collection, and efficiency of data between the paper and electronic Quick Reference Sheets. There are four null hypotheses (H1-H4) and corresponding alternative hypotheses (H1a-H4a):

H1: There is no difference between the Electronic and Paper-Based Quick Reference Sheet on ease of completing in route to the hospital.

H1a: The Electronic Report is easier to complete in route to the hospital.

H2: There is no difference between the Electronic and Paper-Based Quick Reference Sheet on having fields for recording all necessary information.

H2a: The Electronic Report has different fields for recording all necessary information. 
H3: There is no difference between the Electronic and Paper-Based Quick Reference Sheet being a convenient way of collecting patient information.

H3a: The Electronic Report is a more convenient way of collecting patient information.

H4: There is no difference between the Electronic and Paper-Based Quick Reference Sheet on efficiency of delivering patients' information to the hospital.

H4a: The Electronic Report is a more efficient method of delivering patients' information to the hospital.

\section{PROTOTYPE DESIGN}

\section{Prototype Design: Universal Windows Platform}

Currently, more people access the internet through a mobile/smart phones rather than a desktop computer (Qin, Tang, Jang, \& Lehto, 2017). Keeping the advancements in mobile applications and smart phone technology in mind, this research study proposes to move from paper-based medical form approach to digital-based application (Windows based). The Windows platform was selected because even through Android and iOS are leading mobile operating systems in the market (StatCounter, 2017b), when it comes to desktop, windows is still far ahead of all of its competitors (StatCounter, 2017a). Additionally, it was also considered that most emergency technicians have a laptop/tablet with them in an ambulance that is Windows-based. Lastly, taking into consideration the possibility than an EMT might need to use an electronic form on various devices such as mobile to desktop or to tablet; the Windows operating system provides the largest amount of flexibility.

The responses from the EMS staffs reinforced the decision to use the Windows operating system. To fulfill the requirements of EMS, the Universal Windows Platform (UWP) was selected. UWP provides the power to design the application that is suitable to run on any windows-based devices, from a phone to a tablet or a PC (Mike Jacobs, 2017). Apart from the EMS requirements, there were also other advantages of the UWP such as effective pixels and scaling, smart interactions, universal input, controls, and styles and write once and run on any Windows 10 platform.

\section{Prototype Design: Electronic Quick Reference Sheet}

All the emergency services use some kind of paper form to collect patients' information, which first responders later must submit, to the hospital when handing off the patient. Several problems were identified with paper-based sheets. For example, there is the possibility of illegible handwriting (due to either personal style or jarring roads) and lost and or destroyed paper copies. To overcome those problems, an electronic Quick Reference Sheet was designed. One of the main challenges of the paper Quick Reference Sheets is that there is no standard template or format. When discussed with several different EMS representatives from different counties, we observed that they all were using different names such as quick reference sheet, 60-second sheet, etc. Even the template was different; e.g.: one had options to check medication given while the others did not, or in treatment section one would just have a line for writing everything down and the others had listed options where only specific data need to be added. Some of these disparities might affect EMTs transferring from one place to another.

To help overcome these issues, an application, which could be used across all EMS providers, was designed. Figure 1 illustrates the electronic Quick Reference Sheet. The top of the form contains the information about EMTs, their current EMS unit, the call run number, the current date, and the reason of the call. The date field automatically takes the system current date so if the application is used in different time zone it will be updated to the current system date accordingly. This option reduces the time taken by EMS providers to complete the form and it allows them to designate more attention to the patient. The next part beneath it, to the left shows the personal information of the patient. It contains the basic demographics like name, address, date of birth, and social security number. 


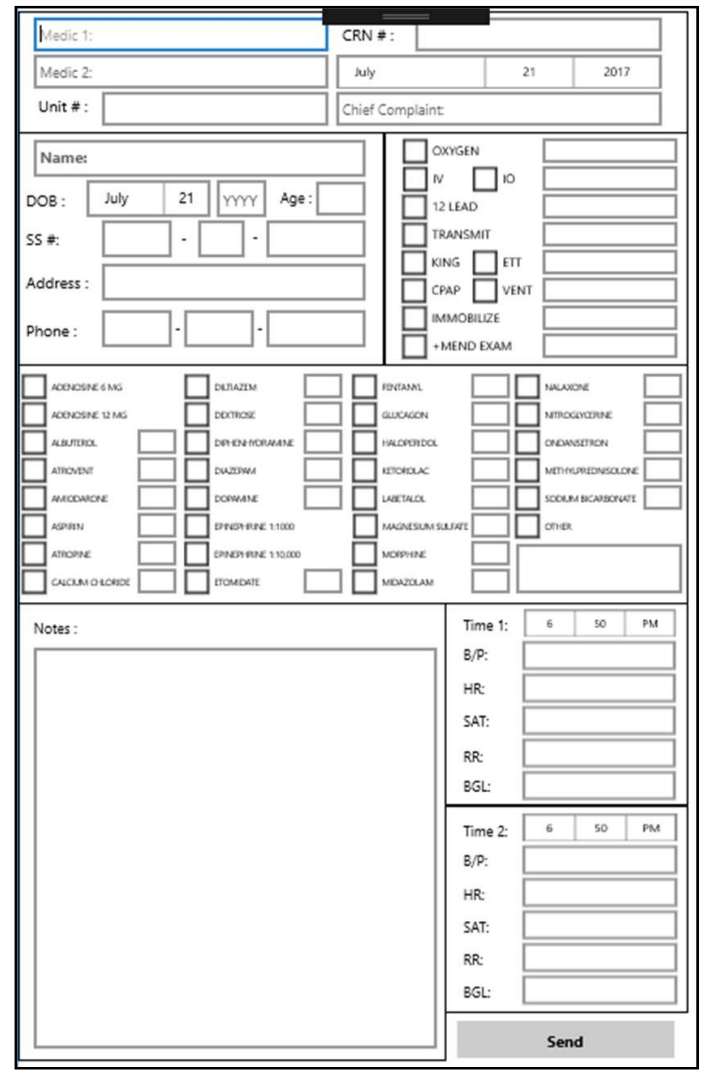

Figure 1. Electronic Quick Reference Sheet

When EMTs were interviewed, they expressed the need of having a phone number field added to the form, as well as age. These suggestions were taken seriously with the addition of fields and functionality was enhanced. For example, age calculator automatically displays the age of the patient when date of birth is entered. This feature can help medical practitioners make a faster decision on the amount of medication dosage needed for the patient. In addition, when choosing the month and day, scroll option is available for faster selection.

The next two sections contain treatments and given medications. Besides the name of the medication, space is added for notating the amount of given medication to the patient. This could also help the doctors for the quick overview of medication dosage given to the patient. The bottom right part contains the vitals measured by the EMTs. They usually notate it twice including the time when the interventions were done. The prototype provides an option of scrolling down for selecting corresponding time. The last section is "notes" - where EMTs can put any other additional information that they think is important for the doctors to know about the patient. The good thing about this section in the electronic version is that the text does not even have to be typed up. A person can just talk to the application and system will automatically write it down.

Once all the information is entered, the form is ready to be submitted. When the "send" button is clicked/tapped, a pop-up window will display with an option to reconfirm submission. This is illustrated in Figure 2. If the "No" option is picked, the application will bring back the form and allow adding additional or missing information. In case "Yes" is selected, the form will be submitted and all the fields will be cleared out so that the form is ready for the next call. After clicking on "Yes", a "Form is sent" pop-up message will display and the hospital will receive that information instantly without having to wait on an ambulance to actually arrive in there physically. EMTs and other emergency personnel will be able to see all the information and interventions done on the patient and can anticipate the arrival. This may speed up the check-in process, handoff and most likely improve patients' outcomes. 


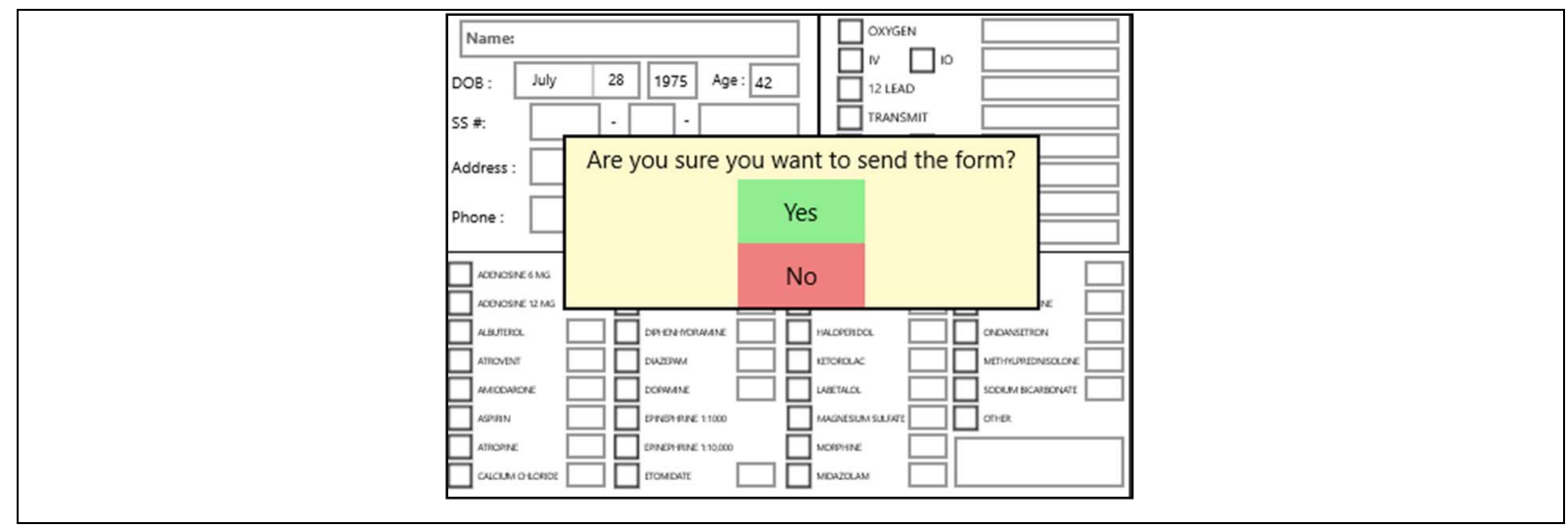

Figure 2. Send Form pop-up

For implementation of this application, Visual Studio 2017 was used. The software was selected for its ease of use, functionality and flexibility. This software includes different languages, which incorporate GUI as well as code in one single package unlike other programming languages like Java, Python, etc. The prototype had set length-limits for phone number and social security fields and all the fields were set to be cleared once the form was submitted. At the end of the code, social security and phone number fields were set to auto-advance.

\section{RESEARCH METHODOLOGY}

The research utilized a questionnaire. Each subject was measured twice. First, EMT's perceptions of the paper-based report were measured. Next the EMT's perceptions on the electronic quick reference sheet were measured All participant answered based on a seven-point Likert scale ranging from strongly disagree to strongly agree. The questionnaire contained four questions regarding the opinions on both, paper and electronic Quick Reference Sheet. Listed below are four of the questions asked related this this research.

1. In my opinion, the Paper/Electronic Quick Reference Sheet would be easy to complete in route to the hospital.

2. In my opinion, the Paper/Electronic Quick Reference Sheet has fields for recording all necessary information.

3. In my opinion, the Paper/Electronic Quick Reference Sheet would be a convenient way of collecting patient information.

4. In my opinion, the Paper/Electronic Quick Reference Sheet would be an efficient method of delivering patients' information to the hospital

\section{Hypothesis Testing}

When comparing populations, such as assessing or testing the difference of the means from two samples of data, a good method to evaluate the significance or the difference is hypothesis testing (Dietrich, Heller, \& Yang, 2015). Forming an assertion and testing it with data is the basic concept of hypothesis testing. The common assumption when performing hypothesis testing is that there is no difference between two samples. This assumption is used for conducting a scientific experiment or constructing, so called, null hypothesis $\left(\mathrm{H}_{0}\right)$ and the alternative hypothesis $\left(\mathrm{H}_{\mathrm{a}}\right)$. A hypothesis test can have two outcomes: 1) Either rejecting the null hypothesis and in favor of the alternative (which would mean that there is a difference between two samples) or 2) Not rejecting the null hypothesis. If the null hypothesis is the population means from the two unrelated groups are equal $\left(\mathrm{H}_{0}: \mathrm{u} 1=\mathrm{u} 2\right)$ and alternative hypothesis is the population means are not equal $\left(\mathrm{H}_{\mathrm{a}}: \mathrm{ul} \neq \mathrm{u} 2\right)$; to either reject or accept the alternative hypothesis. The significance level is $\mathrm{p}<0.05$.

When t-test is a data analysis technique of two data sets, it is used for testing a difference between the given samples when variances of the two normal distributions are unknown. The result from the t-test is known as t-statistic. We have to keep in mind that when we are using Student's t-test, it is assumed that distributions of the two populations have unknown but equal variances (Dietrich et al., 2015). Presume $\mathrm{n} 1$ and $\mathrm{n} 2$ samples are independently and randomly selected from two different populations. If each population is normally distributed with the same variance and with 


\section{Issues in Information Systems}

Volume 19, Issue 3, pp. 81-91, 2018

the same mean $(\mu 1=\mu 2)$, then the $\mathrm{t}$-statistic $\mathrm{t}$, in Equation 1 , follows a t-distribution with $\mathrm{n} 1+\mathrm{n} 2-2$ degrees of freedom (df).

Equation 1:

$$
\mathrm{T}=\frac{\bar{x}_{1}-\bar{x}_{2}}{s_{p} \sqrt{\frac{1}{n_{1}}+\frac{1}{n_{1}}}}
$$

The t-test was chosen because to compare the two small groups as it assumes that the two data sets came from distributions with unequal variances and it is used to determine whether the two samples are likely to have come from distributions with equal population means. Two-sample t-test is used when there are distinct subjects in the two samples. The following formula is used to determine the statistic value $\mathrm{t}$ (Equation 2):

Equation 2:

$$
t^{\prime}=\frac{\bar{x}-\bar{y}-\Delta_{0}}{\sqrt{\frac{S_{1}^{2}}{m}+\frac{S_{2}^{2}}{n}}}
$$

The normal distribution, or normality, is one of the most important and widely used distribution in statistics. A normal distribution has a bell-shaped density curve described by its mean $\mu$ and standard deviation $\sigma$. Due to its bell shape, it is also referred as bell curve. The density curve is centered about its mean and is symmetrical. Its spread is determined by its standard deviation. And at a given point $\mathrm{x}$ the height of a normal density curve is given by where pi represents the observed significance level of the Shapiro-Wilk statistic W (Yale, 1997) as Equation 3.

Equation 3:

$$
\frac{1}{\sqrt{2 \pi \sigma^{2}}} e^{\frac{-(x-\mu)^{2}}{2 \sigma^{2}}}
$$

Shapiro-Wilk is a statistical procedure for testing a complete sample for normality. The test statistic is obtained by dividing the square of an appropriate linear combination of the sample order statistics by the usual symmetric estimate of variance (Shapiro \& Wilk, 1965) and detailed as Equation 4.

Equation 4:

$$
W=\frac{\left(\sum_{i=1}^{n} a_{i} z_{(i)}\right)^{2}}{\sum_{i=1}^{n}\left(z_{i}-\bar{z}\right)^{2}}
$$

The W test statistic for normality is defined by (NIST, 2012a) where W is insignificant if the variable's distribution is not different from normal. $\mathrm{W}$ is approximately equal to the correlation between given data and ideal normal scores. When the sample-variable data are perfectly normal (perfect $\mathrm{H}_{0}$ ), $\mathrm{W}$ is equal to 1 . When $\mathrm{W}$ is significantly smaller than 1, then distribution is non-normal and Ha is accepted. Shapiro-Wilk's W-test is recommended for small and medium samples up to $\mathrm{n}=2000$.

Homogeneity of Variance (HOV) is the condition in which all the variables in a sequence have the same finite variance. When HOV is true for a simple statistical or computational approach for analyzing the data, it may be due to a low level of uncertainty in the data. This is also known as homoscedasticity (Dietrich et al., 2015). Levene's test is used to test whether $\mathrm{k}$ samples have equal variances. Equal variances across samples are known as HOV. There are few tests, like ANOVA, that assume the variances are equal across all groups. Levene's test is used to verify that assumption (NIST, 2012b). According to NIST (2012b), given a variable Y with sample size N that is divided into k subgroups, Levene's test statistics, W, is defined as the following Equation 5: 


\section{Issues in Information Systems}

Volume 19, Issue 3, pp. 81-91, 2018

Equation 5:

$$
W=\frac{(N-k)}{(k-1)} \frac{\sum_{i=1}^{k} N_{i}\left(Z_{i}-Z . .\right)^{2}}{\sum_{i=1}^{k} \sum_{j=1}^{N_{i}}\left(Z_{i j}-Z_{i .}\right)^{2}}
$$

If there are violations of homogeneity of variance and normality in the data, Wilcoxon rank-sum test, a different approach comparable to the paired sample t-test, is used. This test (Wilcoxon, 1945) is a nonparametric hypothesis test that checks whether two groups are identically distributed. Because the Wilcoxon rank-sum test does not make any assumptions about the population distribution, it is considered more rigorous than the t-test.

\section{RESULTS}

Demographic information was collected with a majority $(n=10)$ of the sample data $(n=19)$ that have taken the survey were 34 years of age or less. The predominant race was white. Additionally, almost $79 \%$ were males. There were EMTs (49\%), paramedics (48\%) and 1 EMS director (5\%).

The Sharpio-Wilk test was used to examine the normality of our data. As shown in Table 1, there is no significance in any of the comparisons. Table 2 shows results of Levene's test for homogeneity of variances.

\begin{tabular}{|c|c|c|c|c|c|c|c|c|c|c|}
\hline \multicolumn{5}{|c|}{ Table 1. Test of Normality Shapiro-Wilk } & \multicolumn{6}{|c|}{ Table 2. Test of Homogeneity of Variances } \\
\hline & Group & Statistic & df & Sig. & & Levene & $d f$ & & $\mathrm{df}$ ? & $\mathrm{Sig}$ \\
\hline \multirow[t]{2}{*}{ RQ 1} & 1 & 0.647 & 19 & 0.000 & & Statistic & U11 & & a12 & SIg. \\
\hline & 2 & 0.681 & 19 & 0.000 & RQ 1 & 15.771 & & & 36 & 0 \\
\hline \multirow[t]{2}{*}{ RQ 2} & 1 & 0.768 & 19 & 0.000 & RQ 2 & 2.585 & & & 36 & 0.117 \\
\hline & 2 & 0.693 & 19 & 0.000 & RQ 3 & 8.299 & & & 36 & 0.007 \\
\hline \multirow[t]{2}{*}{ RQ 3} & 1 & 0.703 & 19 & 0.000 & RQ 4 & 0.536 & & & 36 & 0.469 \\
\hline & 2 & 0.662 & 19 & 0.000 & & & & & & \\
\hline \multirow[t]{2}{*}{ RQ 4} & 1 & 0.618 & 19 & 0.000 & & & & & & \\
\hline & 2 & 0.6 & 19 & 0.000 & & & & & & \\
\hline
\end{tabular}

Each subject was measured twice. First, EMT's perceptions of the paper-based report were measured. Next the EMT's perceptions on the electronic quick reference sheet were measured. Based on this, typically the correct statistical test is a paired sample t-test. As stated, normality and homogeneity of variance are important assumptions of the paired sample t-test. Based on violations of homogeneity of variance and/or normality in our data (Table 2), it was necessary to take the non-parametric approach comparable to the paired sample t-test, the Wilcoxon Rank-Sum test as proposed by Wilcoxon (1945). Each question has its own table with results as follows.

\begin{tabular}{|lrr|lrr|}
\hline \multicolumn{2}{|c|}{ Table 3. Wilcoxon Signed-Rank Test Results: Research } & \multicolumn{2}{c|}{ Table 4. Wilcoxon Signed-Rank Test Results: } \\
Question 1 & Electronic & Paper & & Electronic & Paper \\
Mean & 6 & 5.211 & Mean & 5.737 & 5.789 \\
Variance & 0.222 & 1.62 & Variance & 1.316 & 0.398 \\
Observations & 19 & 19 & Observations & 19 & 19 \\
Hypothesized & 0 & & Hypothesized & 0 & \\
Mean Difference & 23 & Mean Difference & 28 \\
df & & df & 0.184 \\
t Stat & 2.333 & t Stat & 0.427 \\
P(T<=t) one-tail & 0.01 & P(T<=t) one-tail & 1.701 \\
t Critical one-tail & 1.714 & t Critical one-tail & \\
\hline
\end{tabular}


Issues in Information Systems

Volume 19, Issue 3, pp. 81-91, 2018

\begin{tabular}{|c|c|c|c|c|c|}
\hline \multicolumn{3}{|c|}{$\begin{array}{l}\text { Table 5. Wilcoxon Signed-Rank Test Results: Research } \\
\text { Question } 3\end{array}$} & \multicolumn{3}{|c|}{$\begin{array}{c}\text { Table 6. Wilcoxon Signed-Rank Test Results: } \\
\text { Research Question } 4 \\
\end{array}$} \\
\hline & Electronic & Paper & & tronic & Paper \\
\hline Mean & 6.053 & 5.316 & Mean & 5.842 & 5.316 \\
\hline Variance & 0.275 & 1.339 & Variance & 1.14 & 1.45 \\
\hline Observations & 19 & 19 & Observations & 19 & 19 \\
\hline $\begin{array}{l}\text { Hypothesized } \\
\text { Mean Difference }\end{array}$ & 0 & & $\begin{array}{l}\text { Hypothesized } \\
\text { Mean Difference }\end{array}$ & 0 & \\
\hline $\mathrm{df}$ & 25 & & $\mathrm{df}$ & 35 & \\
\hline t Stat & 2.516 & & t Stat & 2.314 & \\
\hline $\mathrm{P}(\mathrm{T}<=\mathrm{t})$ one-tail & 0.006 & & $\mathrm{P}(\mathrm{T}<=\mathrm{t})$ one-tail & & 0.01 \\
\hline & & 1.708 & t Critical one-tail & & 1.69 \\
\hline
\end{tabular}

When doing a simple analysis of each question compared to its peer, results show that there are significances between paper vs. electronic Quick Reference Sheet.

- H1: As shown in Table 3, research questions 1 has a $p$ value(0.01) that is less than 0.05 which means that null hypotheses is rejected. Moreover, the alternative hypotheses state that the electronic report is easier to complete in route to the hospital.

- $\quad \mathrm{H} 2$ : Table 4 indicates a $\mathrm{p}$ value $(\mathrm{p}=0.427)$ greater than 0.05 . Therefore the null hypotheses is accepted as there is no significant difference between the paper and electronic Quick Reference Sheet on having fields for recording all necessary information. Even though few extra options, rather than fields, were added to electronic version, electronic Quick Reference Sheet was still made to look as close as possible to paper version so it does not represent a big adjustment if used as a new method of gathering patient information.

- H3: As shown in Table 5, the null hypothesis is rejected because the $p$ value $(p=0.006)$ is less than 0.05. Thus, there is a significant difference between the paper and electronic Quick Reference Sheet regarding convenient way of collecting patient information. The electronic Quick Reference Sheet was perceived as a more convenient way of collecting patient information.

- H4: As shown in Table 6, the null hypothesis is rejected because the $\mathrm{p}$ value $(\mathrm{p}=0.01)$ is less than 0.05 . Thus, there is a significant difference between the paper and electronic Quick Reference Sheet on efficiency of delivering patients' information to the hospital.

\section{DISCUSSION, LIMITATIONS AND CONCLUSIONS}

The results of the prototype testing indicate that the electronic Quick Reference Sheet is a better and easier method for EMTs to gather and complete patient information. The results also indicate that it provides a quicker method of communication of patient information to the hospital. This is of particular importance given that time is of the essence in an emergency. The electronic Quick Reference Sheets also make up for the many challenges of the paper version be reducing the possibility of illegible handwriting (due to either personal style or jarring roads) and lost/destroyed paper copies.

These results are promising and more investigation is necessary to minimize challenges if a full prototype system were to be implemented. After all, there are many challenges from transition from a paper-based system to an electronic one. Some problems that may arise from changing from one system to another are: costs of adoption, reluctance of some providers, training procedures, privacy concerns (Ndifon, Edwards, \& Halawi, 2016), as well as time delays during accommodation process, and the need for information technician position openings.

While the results of the prototype were successful, there are limitations that should be addressed. First, the data sample was small. Secondly, this study focused on a comparison of two types of Quick Reference Sheets (paper and 


\section{Issues in Information Systems \\ Volume 19, Issue 3, pp. 81-91, 2018}

electronic). Third, it explored basic usability perceptions and did not deploy a technology acceptance model on the prototype electronic Quick Reference Sheet. Lastly, EMT's were not able to deploy the prototype in a real-world situation. For the security human subject research and safety reasons, this study did not deploy the prototype electronic Quick Reference Sheet in a real-world situation due to federal laws, regulations, and mandatory FDA approval. This last limitation is the most important and provides the strongest impetus for future research.

In conclusion, as the US population ages, EMS will continue to be high priority for the healthcare systems. The need for EMT and ambulance crews to use EMS should be changed as this could provide patient information in seconds, reduce data loss, and miscommunication. This study contributes to the literature by designing and testing a prototype of an electronic Quick Reference Sheet and comparing it to a paper Quick Reference Sheet regarding usage, completion, collection, and efficiency of data. This study found that EMTs perceived electronic Quick Reference Sheets as a better way of collecting the information, easier to complete, and a more efficient way of delivering the information to the hospital. With future research and implementation, this prototype has the possibility and opportunity to make an impactful difference in human lives.

\section{REFERENCES}

Andreasson, S., \& Winge, M. (2010). Innovations for sustainable health and social care-Value-creating health and social care processes: VINNOVA-Verket för Innovationssystem/Swedish Governmental Agency for Innovation System.

Audet, A. M., Squires, D., \& Doty, M. M. (2014). Where are we on the diffusion curve? Trends and drivers of primary care physicians' use of health information technology. Health services research, 49(1pt2), 347360.

Baird, S., \& Boak, G. (2016). Leading change: introducing an electronic medical record system to a paramedic service. Leadership in Health Services, 29(2), 136-150.

Bledsoe, B. E., Wasden, C., \& Johnson, L. (2013). Electronic prehospital records are often unavailable for emergency department medical decision making. Western Journal of Emergency Medicine, 14(5), 482.

Car, J. (2008). The Impact of Ehealth on the Quality \& Safety of Healthcare: A Systematic Overview \& Synthesis of the Literature. Report for the NHS Connecting for Health Evaluation Programme. Extended Executive Summary: University of Edinburgh.

Cuk, S., Wimmer, H., \& Powell, L. M. (2017). Problems associated with patient care reports and transferring data between ambulance and hospitals from the perspective of emergency medical technicians. Issues in Information Systems, 18(4).

Dietrich, D., Heller, B., \& Yang, B. (2015). Data Science \& Big Data Analytics Discovering, Analyzing, Visualizing and Presenting Data pp. 420). Available from http://csis.pace.edu/ctappert/dps/d86015/assign/books/2015DataScience\&BigDataAnalytics.pdf

Finnell, J. T., \& Overhage, J. M. (2010). Emergency medical services: the frontier in health information exchange. Paper presented at the AMIA Annual Symposium Proceedings.

Frellick, M., \& Vega, C. (2016). Can a Web-Based "Handoff" Tool Reduce Medical Errors? Medscape Education Clinical Briefs, from http://www.medscape.org/viewarticle/868002

Hamid, F., \& Cline, T. (2013). Providers' acceptance factors and their perceived barriers to electronic health record (EHR) adoption. Online Journal of Nursing Informatics, 17(3).

Jensen, T. B., \& Aanestad, M. (2006). How healthcare professionals "make sense" of an electronic patient record adoption. Information systems management, 24(1), 29-42. 
Johnson, O. A., Fraser, H. S., Wyatt, J. C., \& Walley, J. D. (2014). Electronic health records in the UK and USA. The Lancet, 384(9947), 954.

Kilmon, C. A., Fagan, M. H., Pandey, V., \& Belt, T. (2008). Using the task technology fit model as a diagnostic tool for electronic medical records systems evaluation. Issues in Information Systems, 9(1), 196-204.

Landman, A. B., Lee, C. H., Sasson, C., Van Gelder, C. M., \& Curry, L. A. (2012). Prehospital electronic patient care report systems: early experiences from emergency medical services agency leaders. PLoS One, 7(3), e32692.

Maillet, É., Mathieu, L., \& Sicotte, C. (2015). Modeling factors explaining the acceptance, actual use and satisfaction of nurses using an Electronic Patient Record in acute care settings: An extension of the UTAUT. International journal of medical informatics, 84(1), 36-47.

Mike Jacobs, A. K., Michael Satran. (2017, 05/19/2017). Introduction to UWP app design. Retrieved 07/16, 2017, from https://docs.microsoft.com/en-us/windows/uwp/layout/design-and-ui-intro

Money, A. G., Atwal, A., Young, K. L., Day, Y., Wilson, L., \& Money, K. G. (2015). Using the Technology Acceptance Model to explore community dwelling older adults' perceptions of a 3D interior design application to facilitate pre-discharge home adaptations. BMC medical informatics and decision making, $15(1), 73$.

Ndifon, L., Edwards, J. E., \& Halawi, L. (2016). Impact of Electronic Health Records On Patient Outcomes. Issues in Information Systems, 17(4), 187.

Newgard, C. D., Zive, D., Jui, J., Weathers, C., \& Daya, M. (2012). Electronic Versus Manual Data Processing: Evaluating the Use of Electronic Health Records in Out-of-hospital Clinical Research. Academic Emergency Medicine, 19(2), 217-227.

NIST. (2012a). Anderson-Darling and Shapiro-Wilk tests. Retrieved 08/27, 2017, from http://www.itl.nist.gov/div898/handbook/index.htm

NIST. (2012b). Levene Test for Equality of Variances. Retrieved 8/26, 2017, from http://www.itl.nist.gov/div898/handbook/eda/section3/eda35a.htm

NSH Confederation. (2008). A vision for emergency and urgent care: the role of ambulance services. London: NHS Confederation.

Poulymenopoulou, M., Malamateniou, F., \& Vassilacopoulos, G. (2014). E-EPR: a workflow-based electronic emergency patient record. Personal and ubiquitous computing, 18(1), 91-100.

Qin, M., Tang, C.-H. H., Jang, S. S., \& Lehto, X. (2017). Mobile app introduction and shareholder returns. Journal of Hospitality and Tourism Management, 31, 173-180.

Rigby, M., Hill, P., Koch, S., \& Keeling, D. (2011). Social care informatics as an essential part of holistic health care: a call for action. International Journal of Medical Informatics, 80(8), 544-554.

Rivard, S., \& Lapointe, L. (2012). Information technology implementers' responses to user resistance: nature and effects. MIS quarterly, 897-920.

Rizk, E. (2010). Holistic care management requires IT integration. Managed Care (Langhorne, Pa.), 19(9), 43.

Shapiro, S. S., \& Wilk, M. B. (1965). An analysis of variance test for normality (complete samples). Biometrika, 52(3/4), 591-611.

Sheikh, A., Jha, A., Cresswell, K., Greaves, F., \& Bates, D. W. (2014). Adoption of electronic health records in UK 


\section{Issues in Information Systems}

Volume 19, Issue 3, pp. 81-91, 2018

hospitals: lessons from the USA. The Lancet, 384(9937), 8-9.

Starmer, A. J., Spector, N. D., Srivastava, R., West, D. C., Rosenbluth, G., Allen, A. D., et al. (2014). Changes in medical errors after implementation of a handoff program. New England Journal of Medicine, 371(19), 1803-1812.

StatCounter. (2017a). Desktop Operating System Market Share Worldwide. Retrieved 07/16, 2017, from http://gs.statcounter.com/os-market-share/desktop/worldwide/

StatCounter. (2017b). Mobile Operating System Market Share Worldwide. Retrieved 07/16, 2017, from http://gs.statcounter.com/os-market-share/mobile/worldwide

Wilcoxon, F. (1945). Individual Comparisons by Ranking Methods. Biometrics Bulletin, 1(6), 80.

Zorab, O., Robinson, M., \& Endacott, R. (2015). Are prehospital treatment or conveyance decisions affected by an ambulance crew's ability to access a patient's health information? BMC emergency medicine, 15(1), 26. 